\title{
Immunoglobulin G4-Associated Cholangitis: One Variant of Immunoglobulin G4-Related Systemic Disease
}

\author{
Yasser A. Alderlieste Bram D.J. van den Elzen Erik A.J. Rauws Ulrich Beuers
}

Department of Gastroenterology and Hepatology, Academic Medical Center, University of Amsterdam, Amsterdam, The Netherlands

\section{Key Words}

Autoimmune pancreatitis · Immunoglobulin G4-related systemic disease $\cdot$ Immunoglobulin G4-associated cholangitis

\begin{abstract}
IgG4-associated cholangitis (IAC) is a recently defined disease entity which shares a number of clinical, biochemical, and radiological features with primary sclerosing cholangitis (PSC). In contrast to PSC, IAC responds to immunosuppressive treatment, is not associated with inflammatory bowel disease, and mainly affects elderly men above the age of 60 years. Today, IAC is regarded as one variant of IgG4-related systemic disease (ISD) of which autoimmune pancreatitis (AIP) is the best studied organ manifestation. The diagnosis of IAC is based on biochemical, radiological and histologic features, among which elevated serum levels of IgG4, extraand intrahepatic biliary strictures as visualized by cholangiography, multifocal IgG4-rich lymphoplasmacytic sclerosing infiltrations in liver and bile duct tissue, and association with AIP are of key importance. This review aims at summarizing clinical features, diagnostic criteria, therapeutic strategies and most recent insights in the pathophysiology of IAC and other organ manifestations of ISD.
\end{abstract}

Copyright $\odot 2009$ S. Karger AG, Base (c) 2009 S. Karger AG, Basel

0012-2823/09/0794-0220\$26.00/0

Fax +41613061234 E-Mail karger@karger.ch www.karger.com

\section{Concept of IgG4-Related Systemic Disease}

The first case reports of IgG4-related systemic disease (ISD)-like disorders date back to the sixties [1]. In 1984, Montefusco et al. [2] described a series of case reports of patients with combined pancreatic and extra-pancreatic disease. The predominant extrapancreatic feature noted was 'sclerosing cholangitis with primary sclerosing cholangitis (PSC)-like features'. In 1999, Erkelens et al. [3] described 4 cases of sclerosing cholangitis with good response to corticosteroid therapy, therefore posing the idea of a 'presumable autoimmune basis' for this type of sclerosing cholangitis.

The pancreatic manifestation is by far the best studied variant of ISD. Yoshida et al. [4] proposed the entity of autoimmune pancreatitis (AIP) characterized by typical biochemical and radiological features and a unique response to therapeutic intervention. They, and subsequently others, found that these patients had elevated levels of serum IgG, particularly of the IgG4 subtype, diffuse irregular narrowing of the main pancreatic duct, massforming pancreatic lesions, IgG4-rich lymphoplasmacytic sclerosing infiltrates in pancreatic tissue, and a favorable response to treatment with corticosteroids, suggestive of an autoimmune response. Remarkably, IgG4-rich lymphoplasmacytic infiltrates appeared not to be limited to the pancreas as these infiltrates could be demonstrated in a variety of other organs in AIP patients, suggesting a systemic disorder characterized by these IgG4-rich lymphoplasmacytic infiltrates.
Prof. Dr. Ulrich Beuers

Department of Gastroenterology and Hepatology, G4-213

Academic Medical Center, University of Amsterdam

PO Box 22700, NL-1100 DE Amsterdam (The Netherlands)

Tel. +31 20566 2422, Fax +31 20691 7033, E-Mail u.h.beuers@amc.uva.nl 
Based on all these findings, Kamisawa et al. [5], in 2003, coined the term ISD for this novel clinicopathological entity. The concept of an ISD has since then been confirmed by many reports on multiorgan inflammatory, mass-forming lesions, sharing typical histological features and corticosteroid-responsiveness [6-11] now considered characteristic of ISD.

Despite the increasing recognition of ISD as an independent disease entity, its pathogenesis still remains largely unknown. To provide an actual overview on this intriguing disease with a focus on its hepatobiliary manifestation, and to further enhance clinical awareness for ISD, we reviewed recent literature. To illustrate the diagnostic challenges and dilemma's to recognize IgG4-associated cholangitis (IAC) at first clinical presentation, we also describe 2 recent cases typical of IAC within the entity of ISD.

\section{Case Reports}

\section{Case 1}

A 76-year-old man presented in March 2007 with jaundice, general malaise and weight loss, without any pain. On a computed tomography (CT) scan, a pancreatic head tumor was seen, and endoscopic retrograde cholangiopancreaticography (ERCP) revealed dilatation of the biliary tree, with a distal stenosis of the common bile duct, but no other bile duct abnormalities. A malignant pancreatic head tumour was strongly suspected, and pancreaticoduodenectomy (Whipple procedure) was performed. Histological investigation of the excised pancreatic tissue, however, revealed a fibrosing inflammation without any signs of malignancy. A few weeks after surgery, the patient presented with recurrence of jaundice. A percutaneous transhepatic cholangiography revealed marked intrahepatic bile duct irregularities with focal, PSC-like stricturing and dilatations, clearly different from the initial pre-operative cholangiography. There was no evidence of anastomotic dysfunction of the hepaticojejunostomy. A liver biopsy revealed marked cholestasis, but no characteristic signs of PSC. The suspicion of AIP and IAC was confirmed by an elevated serum IgG4 of $12.5 \mathrm{~g} / \mathrm{l}$ (normal $<1.4 \mathrm{~g} / \mathrm{l}$ ), with a total IgG of 24.2 $\mathrm{g} / \mathrm{l}$ (normal $<16 \mathrm{~g} / \mathrm{l}$ ). Retrospectively, IgG4 immunostaining of pancreatic tissue revealed marked periductal lymphoplasmacellular infiltration with IgG4-positive plasma cells ( $>10 / \mathrm{HPF})$ in the pancreatic head. There was also prominent IgG4-positive lymphoplasmacellular infiltration in the wall of the excised gallbladder. The duodenal wall did not contain any IgG4-positive cells. Treatment with prednisolone (initial dose, $40 \mathrm{mg}$ daily) led to rapid improvement of jaundice and serum markers of cholestasis. Pancreatic exocrine insufficiency with steatorrhea was successfully treated with oral supplementation of pancreatic enzymes, and a corticosteroid-associated diabetes was controlled with diet and metformin. Eighteen months after the diagnosis of IAC and AIP was made, the patient has regained weight and is clinically in a stable condition and free of complaints. Serum markers of cholestasis have flaired-up intermittently and never normalized completely, and serum IgG4 fluctuates at borderline levels under maintenance immunosuppressive therapy (azathioprine, $50 \mathrm{mg}$ OD, prednisolone, $5 \mathrm{mg} \mathrm{OD}$ ).

Case 2

A 71-year-old man presented with jaundice, fatigue, weight loss and clinical and biochemical signs and symptoms of pancreatic exocrine and endocrine dysfunction. An insulin-dependent diabetes mellitus had been diagnosed a few months before presentation. On CT imaging, a diffusely swollen pancreas was seen, with a tumour in the pancreatic tail. Multiple enlarged lymph nodes were observed in the pancreatic region. On ERCP, an irregular stenosis of $3 \mathrm{~cm}$ was seen in the main pancreatic duct and a contrast stop in the corpus-tail area. Also, a severe irregular stenosis was detected in the intrapancreatic common bile duct, with proximal dilatation, for which a biliary stent was placed. Endoscopic ultrasound (EUS) revealed a mass in the pancreatic corpus, expanding to the pancreatic head and tail. Cytology of multiple fine needle aspirates from the pancreatic mass did not reveal malignant cells. The suspicion of AIP was supported by a serum IgG4 level of $11.9 \mathrm{~g} / \mathrm{l}$. After initiation of oral prednisone, jaundice disappeared and a rapid normalization of cholestatic serum markers and IgG4 was noted, with regression of the pancreatic mass and peripancreatic lymph nodes, in line with a definite diagnosis of AIP according to the HISORt criteria (see below). Corticosteroid treatment was slowly tapered down and was finally stopped. After 6 months, jaundice and fatigue recurred. Serum markers of cholestasis and IgG4 were again elevated. On ultrasonography, variably sized hilar and intrahepatic bile ducts were noted. A liver biopsy was consistent with long-standing cholestasis, and without evidence of PSC, but IgG4 immunostaining in liver tissue was negative. The patient was treated with prednisone and azathioprine $50 \mathrm{mg}$ OD, with clinical recovery and normalisation of cholestatic parameters and serum IgG4 levels. Prednisone treatment was recently stopped, whereas azathioprine was continued at a low maintenance dose.

\section{Clinical Presentations of IgG4-Related Systemic Disease}

\section{IgG4-Associated Cholangitis and Cholecystitis}

IAC is the recently defined hepatobiliary variant of ISD. It is commonly associated with AIP. Most frequent clinical signs and symptoms at presentation included jaundice (77\%), weight loss (51\%), mild to moderate abdominal pain (26\%), steatorrhea (15\%) and new onset diabetes $(8 \%)$ in the largest cohort of 53 patients so far reported [8].

On cholangiography, both intrahepatic bile duct lesions similar to PSC, and extrahepatic biliary strictures mimicking hilar or distal cholangiocarcinoma or pancreatic cancer, can be found. Distal biliary involvement is described in $68-88 \%$ of AIP patients $[6,8,10,12,13]$ and multifocal biliary strictures were observed in one cohort of AIP patients in 32\%, proximal extrahepatic biliary 
Table 1. IAC and PSC: differences in clinical presentation, immunopathological features and treatment response [10, 14-16, 19]

\begin{tabular}{lll}
\hline & PSC & IAC \\
\hline Age, years & $25-45$ & 65 \\
Gender, male & $65 \%$ & $80 \%$ \\
Response to steroids & - & +++ \\
Association with IBD & +++ & - \\
Association with cholangiocarcinoma & +++ & $?$ \\
Other organ involvement & $?$ & +++ \\
Histological findings & obliterative cholangitis and cirrhosis & abundant IgG4-positive plasma cells \\
Dominant cholangiographic findings & band-like strictures with a beaded & segmental strictures and distal bile duct \\
& appearance & strictures \\
Elevated serum IgG4 & $7-9 \% *$ & around $70 \%$
\end{tabular}

* Of note, as the authors themselves suggest, within their cohort of PSC patients, some of the patients with elevated IgG4 levels might in fact be misdiagnosed IAC patients. IBD = Inflammatory bowel disease.

strictures in $37 \%$ and intrahepatic strictures similar to PSC in $31 \%$ as a sign of IAC [8]. In the same series, 4 additional patients showed intrahepatic strictures due to IAC as an isolated finding without evidence of AIP [8]. Wide variations have also been observed in the same patient, when examined at different time points $[12,14,15]$. Thickening of the gallbladder was noted in $32 \%$ of AIP patients [10], and a dense infiltration of IgG4-positive plasma cells was found in some of the gallbladder specimens.

Liver histology in ISD patients is characterized by lymphoplasmacytic infiltrates within and around bile ducts, and obliterative phlebitis and fibrosis leading to sclerosis of the bile ducts. A portal sclerosing inflammation can be observed, but advanced fibrosis or cirrhosis are rare $[8,10,15-18]$. Numbers of IgG4-bearing plasma cells, mainly found in the portal tracts, are significantly higher than in patients with other hepatopathies (including PSC, primary biliary cirrhosis, autoimmune hepatitis or chronic viral hepatitis), correlating to serum IgG4 levels, and decreasing on corticosteroid treatment $[5,14,17$, 18]. Endoscopic biopsy specimen of biliary epithelium revealed >10 IgG4-positive cells/high power field (HPF) in $88 \%$ of 16 biopsied patients with IAC [8]. Biliary or liver biopsy can thus be supportive in diagnosing IAC in ISD, or differentiating it from PSC or pancreatic or biliary cancer.

Although biliary biopsies might be helpful in the differentiation between IAC and PSC, the clinical differentiation can be challenging. Nishino et al. [15] identified different clinical profiles, comparing 24 patients each with IAC and PSC. On cholangiography, IAC was characterized by more segmental strictures and strictures of the distal bile ducts, compared to the predominant bandlike strictures with a beaded appearance in PSC patients. In liver biopsy specimen, fibrous obliterative cholangitis was observed in PSC only, whereas abundant IgG4-positive plasma cell infiltration was typical of IAC patients. Progression to liver failure or development of biliary cancer only occurred in 8 and 3 PSC patients, respectively. Highly significant differences between IAC and PSC in age of onset, occurrence of diabetes mellitus, inflammatory bowel disease and salivary gland swelling, as well as elevated serum IgG4 levels have also been reported by others $[10,14-16,19]$, as summarized in table 1 . Interestingly, 7-9\% of PSC patients in large cohorts had elevated serum IgG4 levels at retrospective analysis [14]. Some of these patients might be IAC patients misdiagnosed as PSC.

\section{Autoimmune Pancreatitis}

ISD predominantly affects the pancreas. AIP (as part of ISD) is a disease of elderly men, with average age consistently reported around 65 years, with a male predilection of $80 \%$. The prevalence of AIP or ISD is largely unknown, although retrospective series report AIP in 4 to $6 \%$ of patients with chronic pancreatitis [20]. Main presenting symptoms are obstructive jaundice (around 70\%; due to intrapancreatic biliary obstruction or IgG4-associated sclerosing cholangitis), diabetes mellitus (around 40$60 \%$ ), weight loss and mild abdominal pain ( $\geq 30 \%$, each) $[6,8,10-13,15,16,18,20-23]$. Serum alkaline phosphatase is elevated in up to $100 \%$ of patients with AIP/IAC. None of these signs and symptoms are specific for AIP and most are shared by patients with pancreatic malignancies. Because imaging features in AIP can also typically mimic 
pancreatic cancer, as discussed later, it is not surprising that up to a third of AIP patients are diagnosed after major surgery for suspected malignancy $[8,23,24]$. In North America, about $2.5 \%$ of all pancreatoduodenectomy specimens revealed histological signs of AIP after surgery [9]. Fortunately, increasing awareness for AIP may have contributed to a recent decline in the number of patients diagnosed with AIP only after major surgery [8].

Extrapancreatic manifestations of ISD may precede AIP, may be observed simultaneously or may become evident many years after AIP $[6,8,19,25]$ as discussed below.

\section{IgG4-Related Sclerosing Sialadenitis}

Swelling of the salivary glands was reported in up to a quarter of patients with AIP, improving after steroid therapy. Salivary gland function examined by sialochemistry and salivary gland scintigraphy was also markedly impaired in many patients with AIP. In ISD patients with sialadenitis, the salivary glands show dense infiltration of IgG4-positive plasma cells and fibrosis, similar to histological findings in AIP and IAC. These histologic features were not identified in salivary gland tissue of patients with Sjögren's syndrome, neither were serum levels of IgG4 elevated in these patients. IgG4-related sclerosing sialadenitis must, therefore, be considered as a distinct manifestation of ISD $[10,13,16,19]$.

\section{Gastrointestinal Involvement in ISD}

Gastrointestinal involvement in ISD has been reported. Kamisawa et al. [8,24] described moderate to severe infiltration with IgG4-positive plasma cells $(\geq 10 / \mathrm{HPF})$ in all 8 histological specimen of endoscopically normal-appearing major duodenal papillae of AIP patients in comparison to the rate of IgG4-positive plasma cells ( $\leq 3 / \mathrm{HPF})$ in papillar histological specimen of 10 control patients with other pancreatic pathology or papillitis. The same group earlier described heavy infiltration with IgG4-positive plasma cells $(>10 / \mathrm{HPF})$ in the lamina propria of 7 of 10 stomach biopsies, 2 of 5 colonic biopsies, and 3 of 18 major duodenal papilla biopsies, in a group of 24 patients with AIP, but in none of their control patients [26]. There were no distinct endoscopic findings, though foci of slightly pale, thickened mucosa with loss of visible vascular pattern were observed in some cases [26]. In contrast, Shinji et al. [27] found gastric ulcers with IgG4-positive plasma cells on histology in 8 of 23 selected AIP patients. These studies are highly suggestive of gastrointestinal involvement in ISD, and indicate that mucosal infiltration with $\geq 10$ IgG4-positive plasma cells/HPF may be rather spe- cific (although not that sensitive) for the diagnosis of ISD. Prospective cross-validation of these findings in independent cohorts of patients with ISD is certainly needed.

\section{Retroperitoneal Fibrosis in ISD}

Retroperitoneal fibrosis was reported in association with AIP in $3-10 \%$ of patients, resolving after corticosteroid therapy. Dense infiltration of IgG4-positive plasma cells and obliterative phlebitis in fibrotic lesions in the retroperitoneum were described $[10,13,16,21]$.

\section{Lymphadenopathy in ISD}

Abdominal lymphadenopathy with dense infiltration of IgG4-positive plasma cells was detected on laparotomy in 5 of 8 patients with AIP [16]. Cervical or mediastinal lymphadenopathy was observed in the same study on CT scan in 7 of 28 AIP patients, disappearing after corticosteroid therapy.

\section{Pulmonary Involvement in ISD}

Increased uptake in gallium-67 scintigraphy, suggestive of pulmonary hilar involvement, was described in $80 \%$ of a large cohort of patients with AIP [10]. In another study, pulmonary involvement was reported in 4 of 30 patients with AIP, showing good response to corticosteroid therapy [16].

\section{Renal Involvement in ISD}

There are various case reports on renal involvement in patients with AIP [7, 12, 13, 20]. Takahashi et al. [13] described small peripheral cortical nodules or diffuse patchy areas in renal parenchyma suggestive of renal involvement on CT or MRI in 35\% of a group of 40 patients with AIP which regressed after corticosteroid treatment. Serum creatinine levels were not affected in these patients.

\section{Other Manifestations in ISD}

Other reported manifestations in ISD include IgG4related prostatitis and aortitis, and localizations in the thyroid gland and bone marrow $[7,10,11,28,29]$.

\section{Biochemical Markers in ISD}

\section{Serum IgG4}

Elevated serum immunoglobulin levels were described early in AIP [4]. In 2001, Hamano et al. [30] reported in a landmark study that IgG4 levels $>135 \mathrm{mg} / \mathrm{dl}$ had a sensitivity $>97 \%$ for diagnosing AIP. Since then, numerous 
case series have demonstrated more conservative sensitivities of around $70 \%[7,8,10-14,16,18,21,23,31]$. Serum IgG4 levels may fluctuate over time, and sensitivity is higher when repeated analysis is performed $[7,14]$. In a large cohort of patients with various pancreatic diseases, including 45 patients with AIP, Ghazale et al. [22] reported a specificity of elevated serum IgG4 (>140 mg/dl) of $93 \%$, increasing to $99 \%$ when a cut-off value of $280 \mathrm{mg} / \mathrm{dl}$ was used, thereby reaching a positive predictive value of $75 \%$. Despite remarkable sensitivity and specificity, an elevated serum IgG4 is not regarded as diagnostic for ISD. Elevated serum IgG4 levels (>140 mg/dl) were observed in $10 \%$ of patients with proven pancreatic cancer, as well as in small percentages of patients with acute or chronic pancreatitis [22]. Thus, serum IgG4 can be helpful in supporting the diagnosis of AIP/ISD but is not pathognomonic.

\section{Serum CA19-9}

The established tumor marker, carbohydrate antigen 19-9 (CA19-9), is not a useful marker to discriminate AIP from pancreatic cancer. It was reported to be elevated $(>100 \mathrm{U} / \mathrm{ml})$ in $9-18 \%$ of patients with AIP without evidence of pancreatic cancer in three case series $[8,22,23]$, occasionally with even very high levels.

\section{Serum Auto-Antibodies}

Serum auto-antibodies are often detected in ISD. In various series of AIP patients, antinuclear antibodies (ANA) and rheumatoid factor (RF) were found to be positive in $43-80 \%$ and $13-30 \%$, respectively $[10,11,15,16$, $18,24]$. Anti-smooth muscle and double-stranded DNA antibodies were also occasionally reported $[11,18]$. Thus, these auto-antibodies are not helpful in differentiating AIP from other immune-mediated liver diseases.

Auto-antibodies against carbonic anhydrase (CA-II) and lactoferrin, antigens that are expressed in the ductal epithelial cells of several exocrine glands $[11,16]$, are not specific for ISD and are found in various biliary diseases, but may be helpful in the differentiation of AIP and pancreatic cancer. Hosoda et al. [32] were recently able to demonstrate elevated CA-II antibodies in 8 of 9 AIP cases using an arbitrarily defined ELISA absorbance cut-off value. Remarkably, none of the 32 control patients with pancreatic cancer had elevated CA-II antibodies, thereby yielding $100 \%$ specificity for AIP compared to pancreatic cancer. These results remain to be confirmed in larger studies.

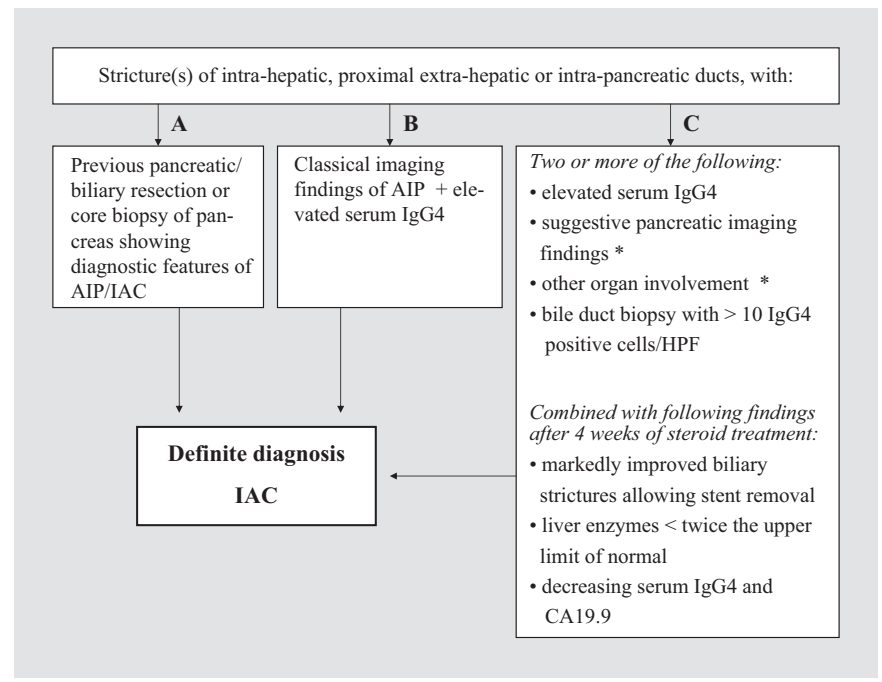

Fig. 1. HISORt diagnostic criteria for IAC [8]. * For details, see text.

\section{Diagnosis of ISD and Its Organ Manifestations}

The diagnosis of ISD and its organ manifestations is currently based on a combination of serological (as discussed above), imaging and histologic features.

\section{$I A C$}

IAC presents with biochemical and cholangiographic features frequently indistinguishable from PSC. Ghazale et al. [8] recently proposed a set of diagnostic criteria for diagnosing IAC (fig. 1). This diagnostic recommendation may temporarily serve as a guideline for diagnosing IAC, although it has not yet been cross-validated in an independent cohort of patients with IAC.

\section{AIP}

An accurate diagnosis of AIP and its differentiation from other forms of pancreatitis, but particularly from pancreatic or bile duct cancer is of utmost importance, both to prevent patients with AIP from undergoing unnecessary surgery, and to prevent delayed diagnosis of possible fatal cancer. A classical finding in AIP is the diffusely enlarged relatively hypodense 'sausage-like' pancreas, with delayed enhancement of the pancreatic parenchyma on imaging (CT or MRI). Mild peri-pancreatic lymphadenopathy is commonly seen $[5,9-11,16,17,27$, 31], whereas peri-pancreatic fat infiltration is uncommon. Pancreatic parenchymal calcifications vary between 8 and $14 \%[21,24]$, while pancreatic cysts are rare in AIP [10, 24]. Classical AIP findings in ERCP are focal, segmental, 


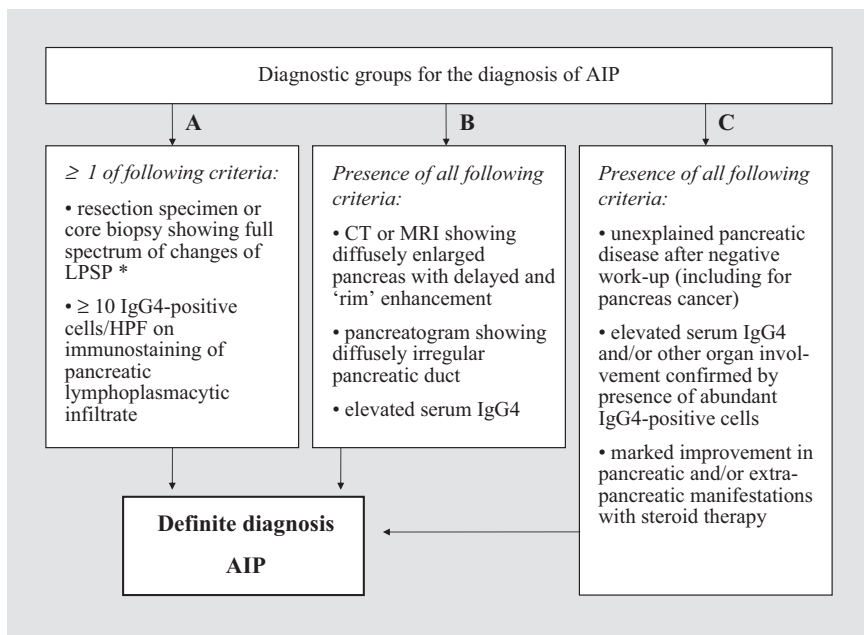

Fig. 2. HISORt diagnostic criteria for AIP [21]. Three diagnostic groups are defined, based on histological features, characteristic imaging findings, or coexisting other findings suggesting the diagnosis. ${ }^{*}$ See text.

or diffuse narrowing of the main pancreatic duct, although many variations exist, depending on the course of disease $[6,8,12,20,23,24]$. On EUS, typically, a hypoechoic gland with focal or diffuse parenchymal swelling can be seen [20]. Fluorodeoxyglucose positron emission tomography (FDG-PET) revealed FDG uptake in the pancreas in AIP patients $[16,33]$ that disappeared after corticosteroid treatment in a small study with 6 patients [34]. Atypical findings at presentation, however, are common in AIP patients [23,24]. They include focal pancreatic enlargements, low-echoic masses, or a double duct sign and occasionally multiple masses or even progression from a localized to a diffuse involvement of the pancreas within the same patient, at different time points [33].

The histopathological hallmark in AIP is the presence of abundant IgG4-positive plasma cells $[6,10,16,20,21$, $35]$. In up to $100 \%$ of AIP cases, $>10$ IgG4-positive cells/ HPF can be seen, in both excision and biopsy specimen of the pancreas $[5,17,21,36]$, even in the absence of elevated serum IgG4 [22]. In 175 tissue specimen of various organs of patients without evidence of ISD, only $1(0.6 \%)$ specimen had positive ( $>10$ IgG4-positive cells/HPF) IgG4 immunostaining [7]. Nevertheless, infiltrates rich of IgG4-positive plasma cells have been reported in pancreas specimen of patients with alcoholic pancreatitis or pancreatic adenocarcinomas [36]. Therefore, caution should be taken to diagnose AIP only on the basis of IgG4-positive plasma cells in pancreatic tissue. Helpful in further discriminating AIP from other pancreatic dis- ease is the histologic finding of lymphoplasmacytic sclerosing pancreatitis (LPSP) which includes dense lymphoplasmacytic infiltrates around small-sized pancreatic ducts, with fibrosis centred around ducts and veins. In contrast, ductal dilatation, stones or proteinaceous plugs, are changes typically associated with other types of chronic pancreatitis, but not with AIP.

Two sets of criteria for the diagnosis of AIP have recently independently been proposed which are based on the original Japan Pancreas Society diagnostic criteria of AIP:

HISORt criteria (histology, imaging, serology, other organ involvement, and response to corticosteroids) [21] define three diagnostic groups, based on histological features, characteristic imaging findings, or co-existing other findings suggestive of AIP (fig. 2).

Kim criteria [37]: (1) Classical imaging findings with diffuse enlargement of the pancreas on CT scan and diffuse or segmental irregular narrowing of the main pancreatic duct in ERP, together with one of the following criteria: (2) elevated levels of IgG or IgG4 or positive autoantibodies; (3) histopathological findings of fibrosis and lymphoplasmacytic infiltration, or (4) association with another autoimmune disease.

Diagnosis of AIP is considered probable if only criterion I and IV are met. Response during a 2-week course of steroid therapy can then prove the diagnosis.

Both sets of criteria include histological, imaging and serological findings as well as responsiveness to corticosteroid treatment. The discriminative strength of a diagnostic treatment with corticosteroids was convincingly demonstrated in a group of 22 patients with atypical imaging findings, where a 2-week corticosteroid treatment period helped to identify all of 7 patients with pancreatic cancer [23]. In HISORt, AIP can be diagnosed based on histological findings only, although histological features of AIP might not be specific as outlined above. Still, HISORt criteria comprise typical and atypical imaging features, enhancing its sensitivity to diagnose the wide spectrum of manifestations of AIP $[11,35]$.

In comparison, the Kim criteria focus on pancreatic imaging thereby minimizing the risk of misdiagnosing pancreatic cancer $[11,35]$. However, sensitivity in diagnosing this heterogeneous spectrum of disease is lower.

There is no international consensus on criteria for AIP so far. Increasing awareness of ISD and detection of more sensitive and specific markers for diagnosing ISD may contribute to improve the diagnostic yield for AIP and ISD in the near future $[7,9,10,12,20,21,23,24]$. 


\section{Pathogenesis of ISD}

\section{Auto-Immune or Allergic?}

The pathogenesis of ISD remains largely unknown. Biochemical and clinical features like elevated serum immunoglobulins and particularly IgG4, serum auto-antibodies and the excellent response to steroid therapy may argue for ISD representing an autoimmune disorder. However, an excellent response to corticosteroid treatment is also observed in allergic disorders, and elevated serum IgE levels have been found in $35 \%$ of AIP patients [18]

The human immune system is controlled by $\mathrm{T}$ helper cells (Th), and regulatory T cells (Tregs). Most autoimmune disorders are characterized by Th1-predominant reactions, associated with elevated levels of interferon- $\gamma$ (IFN- $\gamma$ ) produced by Th1 cells, whilst allergic disorders such as bronchial asthma and atopic dermatitis typically show Th2-type responses mediated by interleukin (IL)-4, IL-5, and IL-13. Tregs are capable of immune suppression by producing regulatory cytokines (IL-10 and tumor growth factor (TGF)- $\beta$ ), and play an important role in the prevention of autoimmune disorders [10, 11, 14, 19]. Cytokine profiles in IAC and other extrapancreatic manifestations of ISD demonstrated significant increases in IL-4, IL-5, IL-13, IL-10 and TGF- $\beta$ mRNA expression, as compared to samples from disease control patients with PSC or PBC [19]. IFN- $\gamma$ was similarly expressed in both groups. Furthermore, prominent infiltrations with cells positive for Foxp3, a transcription factor and marker of Tregs were identified. These data suggest an immunopathogenesis of ISD mediated by a Th2-predominant response to an unknown trigger, with large numbers of Tregs in affected tissues clearly different from autoimmune disorders. Interestingly, IL-4 and IL-10 may direct B-cells to produce IgG4 $[19,38]$. In addition, TGF- $\beta$ is a powerful fibrogenic cytokine $[14,19,39]$ possibly mediating IgG4-positive plasma cell infiltration and periductal fibrosis/sclerosis, two of the most prominent pathological findings in ISD. It remains to be mentioned that an earlier study had shown increased IFN- $\gamma$ levels in ISD patients, more indicative of an autoimmune Th1-predominant immune response [40]. Thus, it remains unclear at present whether a Th1-mediated response (as in autoimmune disorders), or a Th2-predominant allergy-like response $[10,11,14,16,18-20]$ dominates the inflammatory reaction in ISD.

\section{Role of IgG4}

Prominent IgG4 in serum and infiltrating plasma cells are the hallmark of ISD. IgG4 is the least frequent IgG subclass, normally accounting for less than $5 \%$ of the IgG pool $[19,41,42]$. Elevated IgG4 titres are found in both allergic diseases, such as asthma and atopic dermatitis $[14,19]$, as well as in autoimmune diseases, including pemphigus vulgaris, myasthenia gravis, vasculitis and systemic lupus erythematosus [42]. Whether IgG4 plays a role in the pathogenesis of ISD is not clear. IgG4 is able to activate the classical complement pathway, it might even have an anti-inflammatory role, by reducing the pool of available antigens to more pathogenic antibodies $[14,19,42,43]$. Nevertheless, IgG4 was found to be a pathogenic auto-antibody in pemphigus vulgaris, capable of inducing dermal-epidermal separation [14, 19, 42]. In rheumatoid arthritis (RA), it was found to be the predominant anti-cyclic citrullinated peptide (CCP) IgG antibody fraction [41]. Serum-IgG4 of ISD patients showed reactivity against biopsy specimen of normal pancreatic and bile duct tissue, suggesting that IgG4 might act as an auto-antibody in ISD [44]. Thus, it remains unclear whether IgG4 auto-antibodies are cause or consequence in ISD.

\section{Treatment of ISD}

\section{$I A C$}

Immunosuppressive treatment has been shown to exert a marked effect on inflammatory activity of IAC, and complete long-term remission after 3 months of treatment has been reported. However, the extent of disease may affect the long-term response, and a retrospective analysis showed that patients with alterations of proximal extrahepatic and intrahepatic bile ducts are prone to a higher risk of relapse after stop of treatment than patients with distal bile duct strictures only (64 vs. 32\%) [8]. Thus, corticosteroids are regarded as the initial treatment of choice in this disease, in similar dosage as in the treatment of AIP (see below). Azathioprine at doses up to $2 \mathrm{mg} / \mathrm{kg} / \mathrm{day}$ should be considered in those with proximal and intrahepatic stenoses and those after relapse during/after corticosteroid therapy. A significant percentage of IAC patients require temporary biliary stenting to resolve cholestasis $[8,12]$. Duration of medical treatment for 3 months may be sufficient for the majority of patients, but long-term maintenance therapy at low doses may be required when disease activity has not completely come to a standstill or has relapsed. The long-term prognosis of IAC is not well known. Cirrhosis and progression to portal hypertension have only occasionally been reported. 


\section{AIP}

The natural course of AIP in ISD is unknown, but some patients seem to improve spontaneously $[12,16]$. Corticosteroids are considered the first line of therapy, providing response in almost all cases. Oral prednisone is usually initiated at $40 \mathrm{mg}$ per day for a period of 1-3 months, followed by a tapering schedule of $5 \mathrm{mg}$ every 1 to 2 weeks $[7,8,10,14,16,20,21]$. Radiological improvement of AIP can occur as early as 1-2 weeks after corticosteroid therapy, with normalization of pancreatic size within a few weeks $[10,23]$. In patients with biliary drainage, stents can be removed in almost all patients after 1 to 2 months $[8,10,14]$. After several weeks of corticosteroid treatment, hypergammaglobulinemia and elevated IgG4 levels can resolve and autoantibodies can become undetectable [20]. Diabetes mellitus also improves following corticosteroid therapy $[10,20]$.

Reports of relapse rates in AIP after corticosteroid withdrawal range from 4 to $53 \%[8,10,12,14,20]$. The most sensitive and early marker for relapse was an increase in serum alkaline phosphatase levels, and increasing serum IgG4 levels after treatment $[8,14]$. Treatment of relapse with a repeated course of corticosteroids and maintenance therapy with low-dose prednisolone (5 mg/day), azathioprine or mycophenolate mofetil was reported, yielding good response rates. Rituximab, a monoclonal antibody directed against the CD20 antigen on B lymphocytes, was reported to be effective in a case of AIP refractory to corticosteroids and 6-mercaptopurine treatment [45].

The long-term prognosis of AIP is obscure. Only sporadic cases of pancreatic malignancies during the course of AIP have been reported [8].

\section{Other Organ Manifestations of ISD}

Like in IAC and AIP, extrahepatic and extrapancreatic lesions in ISD with infiltration of tissues with IgG4positive plasma cells, marked fibrosis and obliterative phlebitis often respond dramatically to corticosteroids $[6,10,11,16]$.

\section{Summary}

ISD and its hepatobiliary and pancreatic manifestations, IAC and AIP, represent a unique disease entity with largely unknown pathogenesis. IAC and AIP respond very well to anti-inflammatory treatment with corticosteroids. AIP is the most common manifestation of ISD, often mimicking pancreatic cancer. Accurate diagnosis is of utmost importance, to prevent unnecessary surgery, while avoiding delayed diagnosis of a potentially fatal malignancy. Awareness of ISD is the key to an accurate diagnosis. Serum IgG4 is currently the easiest available biochemical marker for diagnosing ISD. Elevated levels are characteristic of ISD, and more than a 2-fold elevation was found to be $99 \%$ specific. However, elevated serum levels of IgG4 can be found in patients without ISD, including PSC and pancreatic cancer patients. Tissue IgG4bearing plasma cell infiltrates seem more accurate for the diagnosis, although they can also be observed in pancreatic cancer or chronic pancreatitis. The HISORt diagnostic criteria for IAC and AIP take into account the highly heterogeneous spectrum of manifestations of ISD, the hallmark of diagnosis being IgG4-rich lymphoplasmacytic infiltrates and sclerosis in pancreatic tissue. Biopsy specimens of the stomach, bile duct, major duodenal papilla or liver are easier and safer to obtain, and can thus facilitate the diagnosis of ISD. When ISD is suspected, a probatory treatment with corticosteroids can be discriminative and is an accepted diagnostic criterium. Unraveling the pathophysiological background of ISD remains the key for developing novel diagnostic tests and more specific therapy in the near future.

\section{Acknowledgement} edged.
Clinical support by Dr. M. de Vree is gratefully acknowl-

\section{References}

1 Sarles H, Sarles JC, Muratore R, Guien C: Chronic inflammatory sclerosis of the pancreas: an autonomous pancreatic disease? Am J Dig Dis 1961;6:688-698.

$\checkmark 2$ Montefusco PP, Geiss AC, Bronzo RL, Randall S, Kahn E, McKinley MJ: Sclerosing cholangitis, chronic pancreatitis, and Sjögren's syndrome: a syndrome complex. Am J Surg 1984; 147:822-826.

\footnotetext{
-3 Erkelens GW, Vleggaar FP, Lesterhuis W van Buuren HR, van der Werf SD: Sclerosing pancreato-cholangitis responsive to steroid therapy. Lancet 1999;354:43-44.

4 Yoshida K, Toki F, Takeuchi T, Watanabe S, Shiratori K, Hayashi N: Chronic pancreatitis caused by an autoimmune abnormality: proposal of the concept of autoimmune pancreatitis. Dig Dis Sci 1995;40:1561-1568.
}

5 Kamisawa T, Funata N, Hayashi Y, Eishi Y, Koike M, Tsuruta K, Okamoto A, Egawa N, Nakajima H: A new clinicopathological entity of IgG4-related autoimmune disease. J Gastroenterol 2003;38:982-984.

6 Chari ST: Diagnosis of autoimmune pancreatitis using its five cardinal features: introducing the Mayo Clinic's HISORt criteria. J Gastroenterol 2007;42(suppl 18):39-41. 
7 Deheragoda MG, Church NI, RodriguezJusto M, Munson P, Sandanayake N, Seward EW, Miller K, Novelli M, Hatfield AR, Pereira SP, Webster GJ: The use of immunoglobulin G4 immunostaining in diagnosing pancreatic and extrapancreatic involvement in autoimmune pancreatitis. Clin Gastroenterol Hepatol 2007;5:1229-1234.

-8 Ghazale A, Chari ST, Zhang L, Smyrk TC, Takahashi N, Levy MJ, Topazian MD, Clain JE, Pearson RK, Petersen BT, Vege SS, Lindor K, Farnell MB: Immunoglobulin G4-associated cholangitis: clinical profile and response to therapy. Gastroenterology 2008; 134:706-715.

\9 Kamisawa T, Tu Y, Egawa N, Tsuruta K, Okamoto A: A new diagnostic endoscopic tool for autoimmune pancreatitis. Gastrointest Endosc 2008;68:358-361.

- 10 Kamisawa T, Okamoto A: IgG4-related sclerosing disease. World J Gastroenterol 2008; 14:3948-3955.

11 Okazaki K, Uchida K, Fukui T: Recent advances in autoimmune pancreatitis: concept, diagnosis, and pathogenesis. J Gastroenterol 2008;43:409-418.

-12 Church NI, Pereira SP, Deheragoda MG, Sandanayake N, Amin Z, Lees WR, Gillams A, Rodriguez-Justo M, Novelli M, Seward EW, Hatfield AR, Webster GJ: Autoimmune pancreatitis: clinical and radiological features and objective response to steroid therapy in a UK series. Am J Gastroenterol 2007; 102:2417-2425.

13 Takahashi N, Kawashima A, Fletcher JG, Chari ST: Renal involvement in patients with autoimmune pancreatitis: CT and MR imaging findings. Radiology 2007;242:791-801.

14 Bjornsson E: Immunoglobulin G4-associated cholangitis. Curr Opin Gastroenterol 2008;24:389-394.

-15 Nishino T, Oyama H, Hashimoto E, Toki F, Oi I, Kobayashi M, Shiratori K: Clinicopathological differentiation between sclerosing cholangitis with autoimmune pancreatitis and primary sclerosing cholangitis. J Gastroenterol 2007;42:550-559.

- 16 Kamisawa T, Okamoto A: Autoimmune pancreatitis: proposal of IgG4-related sclerosing disease. J Gastroenterol 2006;41:613-625.

17 Kamisawa T: Immunoglobulin G4-positive plasma cells in organs of patients with autoimmune pancreatitis. Clin Gastroenterol Hepatol 2008;6:715.

-18 Umemura T, Zen Y, Hamano H, Kawa S, Nakanuma Y, Kiyosawa K: Immunoglobin G4hepatopathy: association of immunoglobin G4-bearing plasma cells in liver with autoimmune pancreatitis. Hepatology 2007;46: 463-471.

19 Zen Y, Fujii T, Harada K, Kawano M, Yamada K, Takahira M, Nakanuma Y: Th2 and regulatory immune reactions are increased in immunoglobin G4-related sclerosing pancreatitis and cholangitis. Hepatology 2007; 45:1538-1546.

20 Zandieh I, Byrne MF: Autoimmune pancreatitis: a review. World J Gastroenterol 2007; 13:6327-6332.
21 Chari ST, Smyrk TC, Levy MJ, Topazian MD, Takahashi N, Zhang L, Clain JE, Pearson RK, Petersen BT, Vege SS, Farnell MB: Diagnosis of autoimmune pancreatitis: the Mayo Clinic experience. Clin Gastroenterol Hepatol 2006;4:1010-1016.

22 Ghazale A, Chari ST, Smyrk TC, Levy MJ, Topazian MD, Takahashi N, Clain JE, Pearson RK, Pelaez-Luna M, Petersen BT, Vege SS, Farnell MB: Value of serum IgG4 in the diagnosis of autoimmune pancreatitis and in distinguishing it from pancreatic cancer. Am J Gastroenterol 2007;102:1646-1653.

-23 Moon SH, Kim MH, Park DH, Hwang CY, Park SJ, Lee SS, Seo DW, Lee SK: Is a 2-week steroid trial after initial negative investigation for malignancy useful in differentiating autoimmune pancreatitis from pancreatic cancer? A prospective outcome study. Gut 2008;57:1704-1712.

24 Nakazawa T, Ohara H, Sano H, Ando T, Imai H, Takada H, Hayashi K, Kitajima Y, Joh T: Difficulty in diagnosing autoimmune pancreatitis by imaging findings. Gastrointest Endosc 2007;65:99-108.

25 Kamisawa T, Chen PY, Tu Y, Nakajima H, Egawa N: Autoimmune pancreatitis metachronously associated with retroperitoneal fibrosis with IgG4-positive plasma cell infiltration. World J Gastroenterol 2006;12: 2955-2957.

26 Kamisawa T, Egawa N, Nakajima H, Tsuruta K, Okamoto A, Hayashi Y, Funata N: Gastrointestinal findings in patients with autoimmune pancreatitis. Endoscopy 2005;37: 1127-1130.

27 Shinji A, Sano K, Hamano H, Unno H, Fukushima M, Nakamura N, Akamatsu T, Kawa S, Kiyosawa K: Autoimmune pancreatitis is closely associated with gastric ulcer presenting with abundant IgG4-bearing plasma cell infiltration. Gastrointest Endosc 2004;59:506-511.

28 Sepehr A, Lauwers GY: Gastrointestinal evidence of autoimmune pancreatitis: a rare manifestation. Histopathology 2008; 53: 358-359.

29 Tseng CW, Tsai JJ, Chen CC: Abdominal aortitis associated with autoimmune pancreatitis. Clin Gastroenterol Hepatol 2009;7: e3-e4.

30 Hamano H, Kawa S, Horiuchi A, Unno H, Furuya N, Akamatsu T, Fukushima M, Nikaido T, Nakayama K, Usuda N, Kiyosawa K: High serum IgG4 concentrations in patients with sclerosing pancreatitis. N Engl J Med 2001;344:732-738.

31 Kamisawa T, Tu Y, Nakajima H, Egawa N, Tsuruta K, Okamoto A: Usefulness of biopsying the major duodenal papilla to diagnose autoimmune pancreatitis: a prospective study using IgG4-immunostaining. World J Gastroenterol 2006;12:2031-2033.

32 Hosoda H, Okawa-Takatsuji M, Shinmura W, Hasimoto N, Ozaki Y, Ikeda Y: Potential for differential diagnosis of autoimmune pancreatitis and pancreatic cancer using carbonic anhydrase II antibody. Pancreas 2008; 37:e1-e7.
33 Kajiwara M, Kojima M, Konishi M, Nakagohri T, Takahashi S, Gotohda N, Hasebe T, Ochiai A, Kinoshita T: Autoimmune pancreatitis with multifocal lesions. J Hepatobiliary Pancreat Surg 2008;15:449-452.

34 Nakajo M, Jinnouchi S, Fukukura Y, Tanabe $\mathrm{H}$, Tateno R, Nakajo M: The efficacy of whole-body FDG-PET or PET/CT for autoimmune pancreatitis and associated extrapancreatic autoimmune lesions. Eur J Nucl Med Mol Imaging 2007;34:2088-2095.

35 Kim MH, Kwon S: Diagnostic criteria for autoimmune chronic pancreatitis. J Gastroenterol 2007;42(suppl 18):42-49.

36 Zhang L, Notohara K, Levy MJ, Chari ST, Smyrk TC: IgG4-positive plasma cell infiltration in the diagnosis of autoimmune pancreatitis. Mod Pathol 2007;20:23-28.

37 Kim KP, Kim MH, Kim JC, Lee SS, Seo DW, Lee SK: Diagnostic criteria for autoimmune chronic pancreatitis revisited. World J Gastroenterol 2006;12:2487-2496.

38 Hummelshoj L, Ryder LP, Poulsen LK: The role of the interleukin- 10 subfamily members in immunoglobulin production by human B cells. Scand J Immunol 2006;64:40-47.

39 Detlefsen S, Sipos B, Zhao J, Drewes AM, Kloppel G: Autoimmune pancreatitis: expression and cellular source of profibrotic cytokines and their receptors. Am J Surg Pathol 2008;32:986-995.

40 Okazaki K, Uchida K, Ohana M, Nakase H, Uose S, Inai M, Matsushima Y, Katamura K, Ohmori K, Chiba T: Autoimmune-related pancreatitis is associated with autoantibodies and a Th1/Th2-type cellular immune response. Gastroenterology 2000;118:573581.

41 Engelmann R, Brandt J, Eggert M, Karberg K, Krause A, Neeck G, Mueller-Hilke B: IgG1 and IgG4 are the predominant subclasses among auto-antibodies against two citrullinated antigens in RA. Rheumatology (Oxford) $2008 ; 47: 1489-1492$.

42 Mihai S, Chiriac MT, Herrero-Gonzalez JE, Goodall M, Jefferis R, Savage CO, Zillikens D, Sitaru C: IgG4 autoantibodies induce dermal-epidermal separation. J Cell Mol Med 2007;11:1117-1128.

43 van der Neut KM, Schuurman J, Losen M, Bleeker WK, Martinez-Martinez P, Vermeulen E, den Bleker TH, Wiegman L, Vink T, Aarden LA, De Baets MH, van de Winkel JG, Aalberse RC, Parren PW: Anti-inflammatory activity of human IgG4 antibodies by dynamic Fab arm exchange. Science 2007; 317:1554-1557.

44 Aoki S, Nakazawa T, Ohara H, Sano H, Nakao H, Joh T, Murase T, Eimoto T, Itoh M: Immunohistochemical study of autoimmune pancreatitis using anti-IgG4 antibody and patients' sera. Histopathology 2005;47: 147-158.

45 Topazian M, Witzig TE, Smyrk TC, Pulido JS, Levy MJ, Kamath PS, Chari ST: Rituximab therapy for refractory biliary strictures in immunoglobulin G4-associated cholangitis. Clin Gastroenterol Hepatol 2008;6: 364-366. 